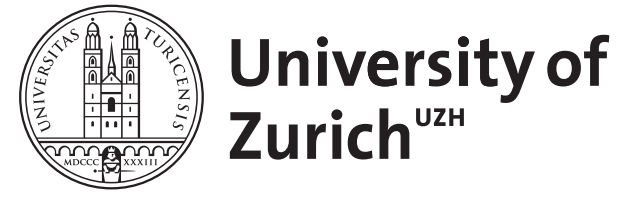

\title{
Delayed diagnosis of tuberculosis
}

\author{
Ruef, C
}

DOI: https://doi.org/10.1007/s15010-010-0069-1

Posted at the Zurich Open Repository and Archive, University of Zurich ZORA URL: https://doi.org/10.5167/uzh-42281

Journal Article

Published Version

Originally published at:

Ruef, C (2010). Delayed diagnosis of tuberculosis. Infection, 38(6):431-432.

DOI: https://doi.org/10.1007/s15010-010-0069-1 


\title{
Delayed diagnosis of tuberculosis
}

\author{
C. Ruef
}

Published online: 4 December 2010

(C) Urban \& Vogel 2010

Tuberculosis remains one of the leading infectious disease threats globally. The pulmonary form of tuberculosis is usually contagious and responsible for the continuous spread of this ancient infectious disease despite intensive public health efforts to reduce the burden of disease in many countries. A primary focus of such efforts is early diagnosis followed by adequate antituberculous treatment.

In this issue of Infection, Verhagen and colleagues [1] report the results of an interesting study on factors that may contribute to a diagnostic delay in tuberculosis patients. The study was conducted in a rural area of Tanzania, and the factors identified by these authors include a failure by the patient to recognize symptoms and a delay by the healthcare system to address the complaints of the patient, draw the appropriate conclusions, perform the necessary tests and, finally, to implement treatment. In an earlier systematic review of the literature, Sreeramareddy [2] reported that of the countries included in his review, Tanzania had the longest total delay in diagnosing tuberculosis. Taking this finding into account, Verhagen and colleagues focused on performing a qualitative survey in this rural area of northwestern Tanzania. Patients were interviewed in a structured fashion, and the researchers even interviewed four traditional healers.

The median total delay to the definitive diagnosis of tuberculosis was 188 days. Only a small fraction of this delay, namely, a median of 21 days, was caused by the patients themselves. The median duration from the first visit to a traditional healer, pharmacy, or dispensary to the first hospital contact was 31 days. Interestingly, most

C. Ruef ( $\square)$

University Hospital of Zurich, Zurich, Switzerland

e-mail: christian.ruef@usz.ch patients did have some basic knowledge of the symptoms of tuberculosis and knew that they should go to a hospital; however, the fear of receiving a diagnosis of human immunodeficiency virus (HIV) infection delayed many from presenting for immediate treatment. In addition, many patients thought their symptoms were caused by malaria. Other reasons for delays on the patients' part were on a spiritual level, as some patients thought their symptoms were caused by witchcraft or were a punishment by God. Over and above the delay caused by reticence of the patients, almost $50 \%$ of the delay could have been avoided if the patients would have presented to the hospital directly rather than first consulting a traditional healer.

The results of this interesting study show that the delay between onset of symptoms and the diagnosis of tuberculosis was to some degree caused by the patients' lack of knowledge of the symptoms of tuberculosis and by spiritual beliefs that interfere with reason. A substantial component of the delay in presenting to a hospital for care was also the desire of patients to first seek help from a traditional healer.

A general principle that can be derived from this study is that future efforts to reduce the delay between the onset of symptoms and the diagnosis of tuberculosis must focus on patient education regarding both the symptoms of tuberculosis and the importance of receiving rapid medical help based on a state-of-the-art diagnostic approach. Whether or not this Western type of approach to the diagnosis of infectious diseases will eventually be accepted by patients living in cultures where traditional healers play an important role remains to be seen. The results of this study suggest that public health efforts need to be enhanced in countries such as Tanzania in order to reduce this diagnostic delay.

C. Ruef, Editor-in-Chief, Infection. 


\section{References}

1. Verhagen LM, Kapinga R, van Rosmalen-Nooijens KAWL. Factors underlying diagnostic delay in tuberculosis patients in a rural area in Tanzania: a qualitative approach. Infection. 2010;38. doi:10.1007/s15010-010-0051-y
2. Sreeramareddy CT, Panduru KV, Menten J, Van den Ende J. Time delays in diagnosis of pulmonary tuberculosis: a systematic review of literature. BMC Infect Dis. 2009;9:91-101. 